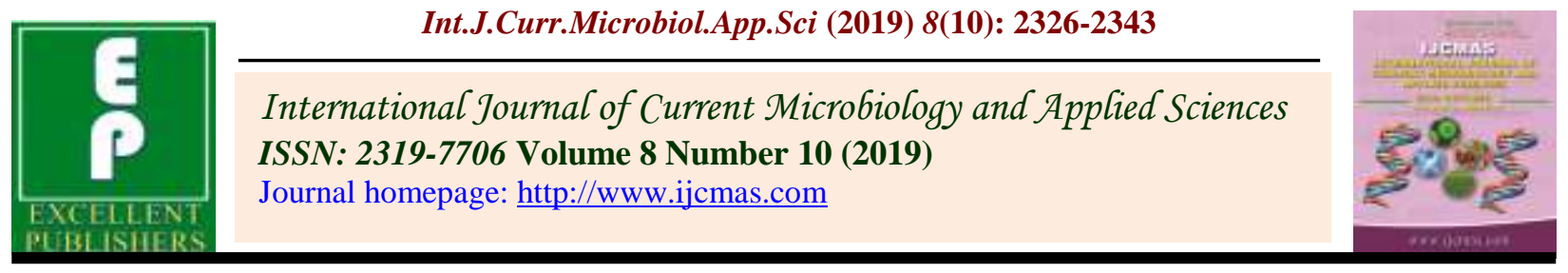

Original Research Article

https://doi.org/10.20546/ijcmas.2019.810.270

\title{
Occurrence and Distribution of Sesame Alternaria Leaf Blight Disease in Nine Agro Climatic Zones of Maharashtra State, India
}

\author{
D.V. Pawar*, A.P. Suryawanshi and V.A. Kadam \\ Department of Plant Pathology, Vasantrao Naik Marathwada Krushi Vidhyapeeth, \\ Parbhani-431 402, Maharashtra, India \\ *Corresponding author
}

\section{Keywords}

Sesame, Alternaria leaf blight disease, Agro climatic zones

Article Info

Accepted:

17 September 2019 Available Online: 10 October 2019

\section{A B S T R A C T}

A roving survey to record sesame Alternaria blight disease intensity was conducted during two Kharif seasons (2014-15 and 2015-16), covering 234 and 240sesame crop fields of nine agro climatic zones, of the Maharashtra state in India, respectively. The results of the survey indicated that overall disease intensity was comparatively higher in the sesame crop grown during Kharif, 2015-16season compared to that of Kharif, 2014-15grown crop. During Kharif, 2014-15 maximum average blight disease intensity of 39.58 per cent was recorded in Central Maharashtra Plateau Zone, followed by the zones viz., Western Maharashtra Plain Zone (37.28 \%), Central Vidarbha Zone (35.19 \%) and Western Maharashtra Scarcity Zone (32.67 \%). In rest of the zones surveyed, the disease intensity was ranged from 21.83 (Western Ghat Zone) to 27.94 (Sub Mountane Zone) per cent. This variability may be due to rainfall pattern, relative humidity, temperature, wind velocity etc. prevailed and a wide range of sesame cultivars grown by the farmers. The pooled mean (Kharif, 2014-15 and 2015-16 ) results revealed maximum mean blight disease intensity in Central Maharashtra Plateau Zone (37.99 \%), followed by the zones viz., Western Maharashtra Plain Zone (36.21 \%), Central Vidarbha Zone (34.27 \%) and Western Maharashtra Scarcity Zone (31.06 \%); whereas, it was minimum in the zones of Western Ghat Zone $(20.24 \%)$ and North Konkan Coastal Zone (21.82 \%). Of various sesame cultivars / varieties grown in the nine agro climatic zones, maximum mean blight disease intensity was recorded on AKT-64 (43.76 \%), followed by Local cultivar (40.08\%); whereas, rest of the cultivars exhibited minimum mean blight disease intensity in the range of 19.63 to 32.33 per cent.

\section{Introduction}

Sesame, (Sesamum indicum L.), is one of the world's ancient important oilseed crop cultivated in more than 55 countries of the world belonging to the family Pedaliaceae and native of India (Joshi,1961; Weiss, 1983). It is an herbaceous, annual, self pollinated (considerable insect cross pollination) crop with $2 \mathrm{n}=26$ chromosomes and which is mentioned in Atharva Veda, Ashtadavaiji, Manusmriti and Puranas as tila indicating the presence of sesame in India since the preChristian era. The world taila, a Sanskrit word 
for oil supposed to have been derived from tila, Sanskrit name of sesame (Yertmanos, 1980). The Vedic scriptures (300-600 BC) contain instructions for using sesame seeds in a ceremonial food, symbolic of immortality. India is among the largest vegetable oil economies in the world and it is ranks first in area and production among the sesame growing countries (Ashri, 1998).

Among the major factors responsible for lower yields, diseases play an important role. Sesame is also affected by many biotic and abiotic stresses. Among the biotic agents, fungi cause major diseases, followed by bacteria, viruses and nematodes. Major Sesame diseases caused by fungi are: Leaf spot / blight (Alternaria sesami), Cercospora leaf spot (Cercospora sesami), Wilt (Fusarium oxysporum $f$. sp. sesami), Root rot (Rhizoctonia bataticola), Powdery mildew (Sphaerotheca fuliginia), stem blight (Phytophthora parasitica) and Anthracnose (Colletotrichum capsici); bacterial Leaf blight / spot (Pseudomonas sesami); Mycoplasmal such as phyllody (Mycoplasma); viral diseases such as Leaf curl (Nicotina virus 10), Mosaic (Cucumber mosaic virus), Necrosis (Tobacco streak virus) and root knot (Meloidogyne hapla) nematode (Bhale et al., 1998). Among these diseases, at present leaf blight caused by Alternaria sesame (Kawamura) is widespread and have continued to be the major constraints in the production and productivity of sesame all over the country in general as well as in the state of Maharashtra particularly. The disease was first reported by Dey (1948) from Uttar Pradesh, which later spread in sesame growing region of the country (Dolle, 1981; Kolte, 1985; Narute and Utikar, 1994; Tripathiet.al, 2005). The disease (A. sesami) has been reported to cause 20 to 40 per cent yield losses all over India (Kumar and Mishra, 1992; Prasad and Reddy, 1997). During Kharif 2014-15, occurrence of Alternaira leaf blight disease was noticed in severe form in farmers fields in different Agro climatic zones of
Maharashtra. Surveys were carried for disease intensity of Alternaira leaf blight in famers' fields. The disease intensity of the disease in commonly grown cultivars was recorded along with variation in Symptoms.

\section{Materials and Methods}

\section{Survey}

A roving survey of randomly selected sesame crop fields covering nine Agro-climatic zones viz., South Konkan Coastal Zone, North Konkan Coastal Zone, Western Ghat Zone, Sub Mountain Zone, Western Maharashtra Plain Zone, Western Maharashtra Scarcity Zone, Central Maharashtra Plateau Zone, Central Vidarbha Zone and Eastern Vidarbha Zone (Fig. 1) of the Maharashtra state was undertaken during Kharif seasons of 2014-15 and 2015-16, to assess Alternaria blight disease intensity and simultaneously to collect the disease samples. In both the years, the survey was conducted during the month of August, when the sesame crop was mostly at $50 \%$ flowering stage. Sesame growing pockets were identified from the records available at the office of Sub-Divisional Agriculture Officers of the respective districts. Details of sesame crop fields surveyed are given in the Table 2.

In the selected sesame crop fields, 10 plants were randomly selected and recorded the observations on blight disease intensity by applying 0-9 grade disease rating scale (Mayee and Datar, 1986), as detailed in Table 1.

Per cent Alternaria blight disease intensity (PDI) was worked out by applying following formula (McKinney, 1923).

Summation of numerical ratings observed PDI = -------- X 100

No. of leaves / plants observed $\mathrm{x}$ maximum grade 


\section{Results and Discussion}

A roving survey to record sesame Alternaria blight disease intensity was conducted during the Kharif seasons of 2014-15 and 2015-16, covering 234 and 240 sesame crop fields, which are distributed under nine Agroclimatic zones of the state of Maharashtra (Table 2). The observations recorded on the overall disease intensity (Table 3), zone-wise intensity (Table 4 and Fig. 1 and 2) and sesame variety-wise intensity (Table 5 and Fig. 3) are presented (PLATE I and II).

Overall and tahsil-wise Alternaria blight intensity in various agro-climatic zones of Maharashtra state

The results obtained on overall per cent Alternaria blight intensity on sesame crop grown in nine agro-climatic zones of Maharashtra covering 63 tahsils each 234 and 240 sesame crop fields, respectively, during Kharif, 2014-15 and 2015-16 seasons is presented in Table 3 and zone-wise range of blight disease intensity in Table 4.

\section{Zone-wise Alternaria blight intensity}

The results (Table 4) revealed that the zonewise sesame blight disease intensity was comparatively higher during Kharif, 2015-16 in the range of 21.83 (Western Ghat Zone) to 39.58 per cent (Central Maharashtra Plateau Zone) with overall average of 29.90 per cent (Fig. 1) than during Kharif, 2014-15 in the range of 18.64 (Western Ghat Zone) to 36.39 (Central Maharashtra Plateau Zone) per cent with overall average of 27.35 per cent. During Kharif, 2014-15 average disease intensity was highest in Central Maharashtra Plateau Zone (36.39\%), followed by the zones viz., Western Maharashtra Plain Zone (35.13\%), Central Vidarbha Zone (33.34 \%), and Western Maharashtra Scarcity Zone (29.44 \%); whereas, in rest of the zones surveyed, the disease intensity was ranged from 18.64 (Western Ghat Zone) to 27.23 (Sub Mountane Zone) per cent.

During Kharif, 2015-16 average disease intensity was ranged from 21.83 (Western Ghat Zone) to 39.58 (Central Maharashtra Plateau Zone) per cent with overall average of 29.90 per cent. However it was maximum in Central Maharashtra Plateau Zone (39.58 \%), followed by the zones viz., Western Maharashtra Plain Zone (37.28 \%), Central Vidarbha Zone (35.19 \%) and Western Maharashtra Scarcity Zone (32.67\%). In rest of the zones surveyed, the disease intensity was ranged from 21.83 (Western Ghat Zone) to 27.94 (Sub Mountane Zone) per cent.

The pooled mean (Kharif, 2014-15 and 201516 ) results (Fig. 2) revealed maximum mean blight disease intensity in Central Maharashtra Plateau Zone (37.99 \%), followed by, Western Maharashtra Plain Zone (36.21\%), Central Vidarbha Zone (34.27 \%) and Western Maharashtra Scarcity Zone (31.06 \%); whereas, it was minimum in the zones of Western Ghat Zone (20.24 \%) and North Konkan Coastal Zone (21.82 \%).

\section{Sesame variety-wise Alternaria blight intensity}

The results (Table 5 and Fig. 3) revealed that in nine agro-climatic zones of the Maharashtra state surveyed, a wide range of sesame varieties / cultivars were grown by the farmers. Due to lack of proven resistance / tolerance against $A$. sesami in the sesame cultivars commonly grown all over the Maharashtra state, all those cultivars are more or less prone to Alternaria leaf spot / blight disease. During Kharif, 2015-16 average disease intensity on sesame varieties was comparatively maximum $(30.85 \%)$ than that of during Kharif, 2014-15 (27.87\%). 
During Kharif, 2014-15 and 2015-16 about 10 varieties of sesame, were grown by the farmers on which average blight disease intensity was comparatively higher (20.64 to $46.39 \%$ ) during Kharif, 2015-16 than that of during Kharif, 2014-15 (18.62 to 41.12 $\%)$. Among the sesame varieties grown, AKT-64 was found to suffer more with the blight intensity of 41.12 and 46.39 per cent, respectively during 2014 and
2015, followed by Local cultivar (39.05 and $41.11 \%)$. Rest of the sesame varieties grown exhibited comparatively minimum to moderate blight intensity the range of 18.62 to 31.00 per cent and 20.64 to 33.65 per cent, respectively during Kharif, 2014-15 and 2015-16. However, the sesame varieties which exhibited minimum average blight intensity were JLT-7 (18.62\% and 20.64 $\%)$, followed by $\mathrm{N}$ -

Table.1 Alternaria blight disease rating scale

\begin{tabular}{|l|l|}
\hline Rating scale & Description \\
\hline $\mathbf{0}$ & $\begin{array}{l}\text { No symptoms on the leaf. } \\
\text { Small, irregular brown spots covering } 1 \text { per cent or less of the } \\
\text { leaf area. }\end{array}$ \\
\hline $\mathbf{3}$ & $\begin{array}{l}\text { Small, round to irregular brown spots with concentric rings } \\
\text { covering 1-10 per cent of the leaf area. }\end{array}$ \\
\hline $\mathbf{5}$ & $\begin{array}{l}\text { Round to irregular brown lesions enlarging, with concentric } \\
\text { rings covering 11-25 per cent of the leaf area. }\end{array}$ \\
\hline $\mathbf{7}$ & $\begin{array}{l}\text { Lesions enlarging and coalescing to form irregular brown } \\
\text { patches with concentric rings and covering } 26-50 \text { per cent of the } \\
\text { leaf area. Lesions also appeared on stem petioles and pods. }\end{array}$ \\
\hline $\mathbf{l}$ & $\begin{array}{l}\text { Lesions enlarged coalesced to forming irregular, dark brown } \\
\text { patches with concentric rings covering } 51 \text { per cent or more of } \\
\text { the leaf area. Lesions on stem petioles and pods. }\end{array}$ \\
\hline
\end{tabular}

Table.2 Agroclimatic zone-wise tahsils and sesame crop fields surveyed in Maharashtra state, during Kharif, 2014-15 and Kharif 2015-16

\begin{tabular}{|l|l|l|c|c|c|c|}
\hline \multirow{2}{*}{ Sr.No. } & \multicolumn{1}{|c|}{ Zone } & \multicolumn{1}{|c|}{ Zones Name } & \multicolumn{2}{c|}{ Kharif2014-15 } & \multicolumn{2}{c|}{ Kharif2015-16 } \\
\cline { 3 - 7 } & & & Tahsils & Fields & Tahsils & Fields \\
\hline $\mathbf{1}$ & I (SKCZ) & South Kokan Coastal Zone & 04 & 13 & 04 & 16 \\
\hline $\mathbf{2}$ & II (NKCZ) & North Kokan Coastal Zone & 03 & 10 & 03 & 09 \\
\hline $\mathbf{3}$ & III (WGZ) & Western Ghat Zone & 03 & 11 & 03 & 10 \\
\hline $\mathbf{4}$ & IV (SMZ) & Sub Mountain Zone & 04 & 15 & 04 & 16 \\
\hline $\mathbf{5}$ & V (WMPZ) & Western Maharashtra Plain Zone & 05 & 20 & 05 & 18 \\
\hline $\mathbf{6}$ & VI (WMSZ) & Western Maharashtra Scarcity Zone & 14 & 52 & 14 & 59 \\
\hline $\mathbf{7}$ & VII (CMPZ) & Central Maharashtra Plateau Zone & 18 & 70 & 18 & 72 \\
\hline $\mathbf{8}$ & VIII (CVZ) & Central Vidarbha Zone & 09 & 34 & 09 & 32 \\
\hline $\mathbf{9}$ & IX (EVZ) & Eastern Vidarbha Zone & 03 & 09 & 03 & 08 \\
\hline Total & & & $\mathbf{6 3}$ & $\mathbf{2 3 4}$ & $\mathbf{6 3}$ & $\mathbf{2 4 0}$ \\
\hline
\end{tabular}


Table.3 Tahsil -wise Alternaria blight disease intensity in the agro-climatic zones of Maharashtra state, during Kharif, 2014-15 and Kharif, 2015-16

\begin{tabular}{|c|c|c|c|c|c|c|}
\hline \multirow[t]{2}{*}{$\begin{array}{l}\text { Sr. } \\
\text { No. }\end{array}$} & \multirow[t]{2}{*}{ Zone } & \multirow[t]{2}{*}{ Tahsils } & \multicolumn{2}{|c|}{ No. of Fields } & \multicolumn{2}{|c|}{$\begin{array}{l}\text { Av. Intensity2 } \\
(\%)\end{array}$} \\
\hline & & & 2014-15 & 2015-16 & 2014-15 & 2015-16 \\
\hline \multirow[t]{4}{*}{1} & \multirow{4}{*}{$\begin{array}{l}\text { I } \\
(\mathrm{SKCZ})\end{array}$} & Vaibhavwadi & 04 & 05 & 22.22 & 27.11 \\
\hline & & Kankavli & 02 & 03 & 20.00 & 24.44 \\
\hline & & Dapoli & 04 & 05 & 18.89 & 18.67 \\
\hline & & Rajapur & 03 & 03 & 23.70 & 28.87 \\
\hline \multicolumn{3}{|c|}{ Overall average } & 13 & 16 & 21.20 & 24.77 \\
\hline \multirow[t]{3}{*}{2} & \multirow{3}{*}{$\begin{array}{l}\text { II } \\
(\mathrm{NKCZ})\end{array}$} & Poladpur & 03 & 03 & 17.04 & 22.96 \\
\hline & & Mahad & 03 & 02 & 19.26 & 18.89 \\
\hline & & Murbad & 04 & 04 & 23.89 & 28.89 \\
\hline \multicolumn{3}{|c|}{ Overall average } & 10 & 09 & 20.06 & 23.58 \\
\hline \multirow[t]{3}{*}{3} & \multirow{3}{*}{$\begin{array}{l}\text { III } \\
(\mathrm{WGZ})\end{array}$} & Chandgad & 04 & 02 & 22.22 & 17.78 \\
\hline & & Patan & 03 & 05 & 14.82 & 26.22 \\
\hline & & Mahabaleshwar & 04 & 03 & 18.89 & 21.48 \\
\hline \multicolumn{3}{|c|}{ Overall average } & 11 & 10 & 18.64 & 21.83 \\
\hline \multirow[t]{4}{*}{4} & \multirow[t]{4}{*}{$\begin{array}{l}\text { IV } \\
(\mathrm{SMZ})\end{array}$} & $\begin{array}{l}\text { Gargoti } \\
\text { (Bhudargad) }\end{array}$ & 02 & 03 & 33.34 & 31.85 \\
\hline & & Karad & 04 & 04 & 28.89 & 22.78 \\
\hline & & Wai & 05 & 05 & 24.44 & 27.11 \\
\hline & & Mulshi & 04 & 04 & 22.22 & 30.00 \\
\hline \multicolumn{3}{|c|}{ Overall average } & 15 & 16 & 27.23 & 27.94 \\
\hline \multirow[t]{5}{*}{5} & \multirow{5}{*}{$\begin{array}{l}\mathrm{V} \\
(\mathrm{WMPZ})\end{array}$} & Shirol & 04 & 02 & 32.22 & 31.11 \\
\hline & & Koregaon & 05 & 05 & 34.22 & 38.67 \\
\hline & & Khandala & 05 & 05 & 39.56 & 39.56 \\
\hline & & Purandar & 03 & 03 & 36.30 & 36.30 \\
\hline & & Sangamner & 03 & 03 & 33.33 & 40.74 \\
\hline \multicolumn{3}{|c|}{ Overall average } & 20 & 18 & 35.13 & 37.28 \\
\hline \multirow[t]{12}{*}{6} & \multirow{12}{*}{$\begin{array}{l}\text { VI } \\
\text { (WMSZ) }\end{array}$} & Sangli & 03 & 03 & 25.92 & 48.15 \\
\hline & & Khanapur-Vita & 03 & 03 & 23.70 & 31.85 \\
\hline & & Atpadi & 02 & 04 & 26.67 & 35.56 \\
\hline & & $\begin{array}{l}\text { Dahiwadi } \\
\text { (Man) }\end{array}$ & 04 & 05 & 30.00 & 28.87 \\
\hline & & Phaltan & 05 & 05 & 31.55 & 36.89 \\
\hline & & Baramati & 04 & 04 & 34.44 & 41.11 \\
\hline & & Sangole & 05 & 05 & 29.78 & 38.67 \\
\hline & & Pandharpur & 04 & 05 & 30.00 & 34.22 \\
\hline & & Shirur & 03 & 02 & 31.85 & 23.34 \\
\hline & & Shrigonda & 02 & 04 & 26.67 & 32.22 \\
\hline & & Rahuri & 04 & 05 & 31.11 & 34.22 \\
\hline & & Vaijapur & 05 & 05 & 32.44 & 31.55 \\
\hline
\end{tabular}




\begin{tabular}{|c|c|c|c|c|c|c|}
\hline & & & & \multicolumn{3}{|c|}{ Contd... } \\
\hline & & Yeola & 05 & 05 & 30.66 & 37.78 \\
\hline & & Dhule & 03 & 04 & 27.41 & 30.00 \\
\hline \multicolumn{3}{|c|}{ Overall average } & 52 & 59 & 29.44 & 32.67 \\
\hline \multirow[t]{18}{*}{7} & \multirow{18}{*}{$\begin{array}{l}\text { VII } \\
\text { (CMPZ) }\end{array}$} & Tuljapur & 02 & 03 & 33.34 & 31.85 \\
\hline & & Nilanga & 04 & 04 & 40.00 & 38.89 \\
\hline & & Udgir & 05 & 05 & 41.33 & 35.11 \\
\hline & & Latur & 05 & 05 & 39.56 & 40.44 \\
\hline & & Ahmadpur & 03 & 04 & 40.74 & 44.45 \\
\hline & & Deglur & 05 & 04 & 36.00 & 42.23 \\
\hline & & Ambajogai & 05 & 05 & 34.22 & 37.78 \\
\hline & & Parli & 05 & 05 & 35.11 & 45.78 \\
\hline & & Gangakhed & 05 & 05 & 29.78 & 32.44 \\
\hline & & Parbhani & 05 & 05 & 38.67 & 43.11 \\
\hline & & Jintur & 03 & 02 & 30.37 & 40.00 \\
\hline & & Partur & 02 & 03 & 37.78 & 36.30 \\
\hline & & Badnapur & 04 & 04 & 25.00 & 37.78 \\
\hline & & Kannad & 03 & 02 & 27.41 & 53.34 \\
\hline & & Jalgaon & 02 & 03 & 33.34 & 27.40 \\
\hline & & Sindkhed Raja & 05 & 05 & 45.78 & 42.22 \\
\hline & & Deulgaon Raja & 05 & 05 & 51.11 & 44.00 \\
\hline & & Amravati & 02 & 03 & 35.56 & 39.26 \\
\hline \multicolumn{3}{|c|}{ Overall average } & 70 & 72 & 36.39 & 39.58 \\
\hline \multirow[t]{10}{*}{8} & \multirow{10}{*}{$\begin{array}{l}\text { VIII } \\
\text { (CVZ) }\end{array}$} & Basmath & 05 & 05 & 37.78 & 29.78 \\
\hline & & AundhaNagnath & 05 & 05 & 35.11 & 35.11 \\
\hline & & Kinwat & 03 & 02 & 31.85 & 40.00 \\
\hline & & Malegaon & 02 & 03 & 28.89 & 37.78 \\
\hline & & Washim & 04 & 04 & 35.56 & 33.33 \\
\hline & & & & & & Contd \\
\hline & & Karanja & 04 & 04 & 36.67 & 35.55 \\
\hline & & Yavatmal & 02 & 02 & 31.11 & 40.00 \\
\hline & & Arvi & 05 & 03 & 29.78 & 27.41 \\
\hline & & Nagpur & 04 & 04 & 33.34 & 37.78 \\
\hline \multicolumn{3}{|c|}{ Overall average } & 34 & 32 & 33.34 & 35.19 \\
\hline \multirow[t]{3}{*}{9} & \multirow{3}{*}{$\begin{array}{l}\text { IX } \\
(\mathrm{EVZ})\end{array}$} & Chimur & 04 & 02 & 19.44 & 21.11 \\
\hline & & Sakoli & 03 & 03 & 23.70 & 31.85 \\
\hline & & Gondia & 02 & 03 & 31.11 & 25.92 \\
\hline \multicolumn{3}{|c|}{ Overall average } & 09 & 08 & 24.75 & 26.29 \\
\hline
\end{tabular}


Table.4 Zone-wise overall average Alternaria blight intensity on sesame crop in Maharashtra state during Kharif, 2014-15 and 2015-16

\begin{tabular}{|c|c|c|c|c|c|c|}
\hline \multirow{2}{*}{$\begin{array}{l}\text { Sr. } \\
\text { No. }\end{array}$} & \multirow[t]{2}{*}{ Zones } & \multicolumn{2}{|c|}{ 2014-15 } & \multicolumn{2}{|c|}{ 2015-16 } & \multirow{2}{*}{$\begin{array}{c}\text { Mean } \\
\text { Intensity } \\
(\%)\end{array}$} \\
\hline & & $\begin{array}{l}\text { No. of } \\
\text { Fields }\end{array}$ & $\begin{array}{c}\text { Intensity } \\
(\%)\end{array}$ & $\begin{array}{l}\text { No. of } \\
\text { Fields }\end{array}$ & $\begin{array}{c}\text { Intensity } \\
(\%)\end{array}$ & \\
\hline 1 & I (SKCZ) & 13 & 21.20 & 16 & 24.77 & 22.99 \\
\hline 2 & II (NKCZ) & 10 & 20.06 & 09 & 23.58 & 21.82 \\
\hline 3 & III (WGZ) & 11 & 18.64 & 10 & 21.83 & 20.24 \\
\hline 4 & IV (SMZ) & 15 & 27.23 & 16 & 27.94 & 27.59 \\
\hline 5 & V (WMPZ) & 20 & 35.13 & 18 & 37.28 & 36.21 \\
\hline 6 & VI (WMSZ) & 52 & 29.44 & 59 & 32.67 & 31.06 \\
\hline 7 & VII (CMPZ) & 70 & 36.39 & 72 & 39.58 & 37.99 \\
\hline 8 & VIII (CVZ) & 34 & 33.34 & 32 & 35.19 & 34.27 \\
\hline 9 & IX (EVZ) & 09 & 24.75 & 08 & 26.29 & 25.52 \\
\hline \multicolumn{2}{|c|}{ Overall average } & 234 & 27.35 & 240 & 29.90 & - \\
\hline
\end{tabular}

Table.5 Sesame variety-wise average Alternaria blight intensity in various agro-climatic zones of Maharashtra state during Kharif, 2014-15 and 2015-16

\begin{tabular}{|c|c|c|c|c|c|}
\hline \multirow[t]{2}{*}{ Varieties } & \multicolumn{2}{|c|}{ 2014-15 } & \multicolumn{2}{|c|}{ 2015-16 } & \multirow{2}{*}{$\begin{array}{c}\text { Pooled } \\
\text { Mean } \\
\text { Intensity } \\
(\%)\end{array}$} \\
\hline & $\begin{array}{l}\text { No. of } \\
\text { Fields }\end{array}$ & $\begin{array}{c}\text { Av. Intensity } \\
(\%)\end{array}$ & $\begin{array}{l}\text { No. of } \\
\text { Fields }\end{array}$ & $\begin{array}{c}\text { Av. Intensity } \\
(\%)\end{array}$ & \\
\hline Local & 63 & 39.05 & 88 & 41.11 & 40.08 \\
\hline AKT-64 & 18 & 41.12 & 16 & 46.39 & 43.76 \\
\hline Gujrat-til-3 & 24 & 26.67 & 13 & 30.94 & 28.81 \\
\hline $\mathrm{N}-8$ & 11 & 19.80 & 12 & 22.78 & 21.29 \\
\hline AKT-101 & 15 & 23.70 & 11 & 25.25 & 24.48 \\
\hline JLT-26(Padma) & 10 & 24.22 & 16 & 26.66 & 25.44 \\
\hline PKV-NT-11 & 18 & 25.92 & 12 & 29.63 & 27.78 \\
\hline JLT-7 (Tapi) & 15 & 18.62 & 21 & 20.64 & 19.63 \\
\hline Phule-til-1 & 29 & 28.20 & 23 & 31.40 & 29.80 \\
\hline JLT-408 & 31 & 31.00 & 28 & 33.65 & 32.33 \\
\hline Average & 234 & 27.87 & 240 & 30.85 & - \\
\hline
\end{tabular}

Av : Average, No : Number 
Table.6 Sesame leaf blight symptomatic variability observed during survey

\begin{tabular}{|c|c|c|c|c|c|}
\hline \multirow{2}{*}{$\begin{array}{l}\text { Sr. } \\
\text { No. }\end{array}$} & \multirow[b]{2}{*}{ Zone No } & \multicolumn{4}{|c|}{ Leaf blight/spot characters } \\
\hline & & Shape & Size & Colour & $\begin{array}{c}\text { Concentric } \\
\text { rings }\end{array}$ \\
\hline 1 & I (SKCZ) & Circular & Small to large & Light gray & Present \\
\hline 2 & II (NKCZ) & $\begin{array}{l}\text { Circular to } \\
\text { irregular }\end{array}$ & Large & $\begin{array}{l}\text { Light brown to } \\
\text { dark brown }\end{array}$ & Absent \\
\hline 3 & III (WGZ) & $\begin{array}{l}\text { Circular to } \\
\text { irregular }\end{array}$ & Small & Light brown & Absent \\
\hline 4 & IV (SMZ) & $\begin{array}{l}\text { Circular to } \\
\text { irregular }\end{array}$ & Medium & $\begin{array}{l}\text { Light brown to } \\
\text { dark brown }\end{array}$ & Absent \\
\hline 5 & V (WMPZ) & Irregular & Large & $\begin{array}{l}\text { Light brown to } \\
\text { dark brown }\end{array}$ & Absent \\
\hline 6 & VI (WMSZ) & $\begin{array}{l}\text { Circular to } \\
\text { irregular }\end{array}$ & Medium & Light brown & Present \\
\hline 7 & VII (CMPZ) & Irregular & Large & Light gray & Absent \\
\hline 8 & VIII (CVZ) & Circular & Medium & $\begin{array}{l}\text { Dark brown to } \\
\text { black }\end{array}$ & Present \\
\hline 9 & IX (EVZ) & Irregular & Small to large & $\begin{array}{l}\text { Light brown to } \\
\text { dark brown }\end{array}$ & Present \\
\hline
\end{tabular}

Plate I
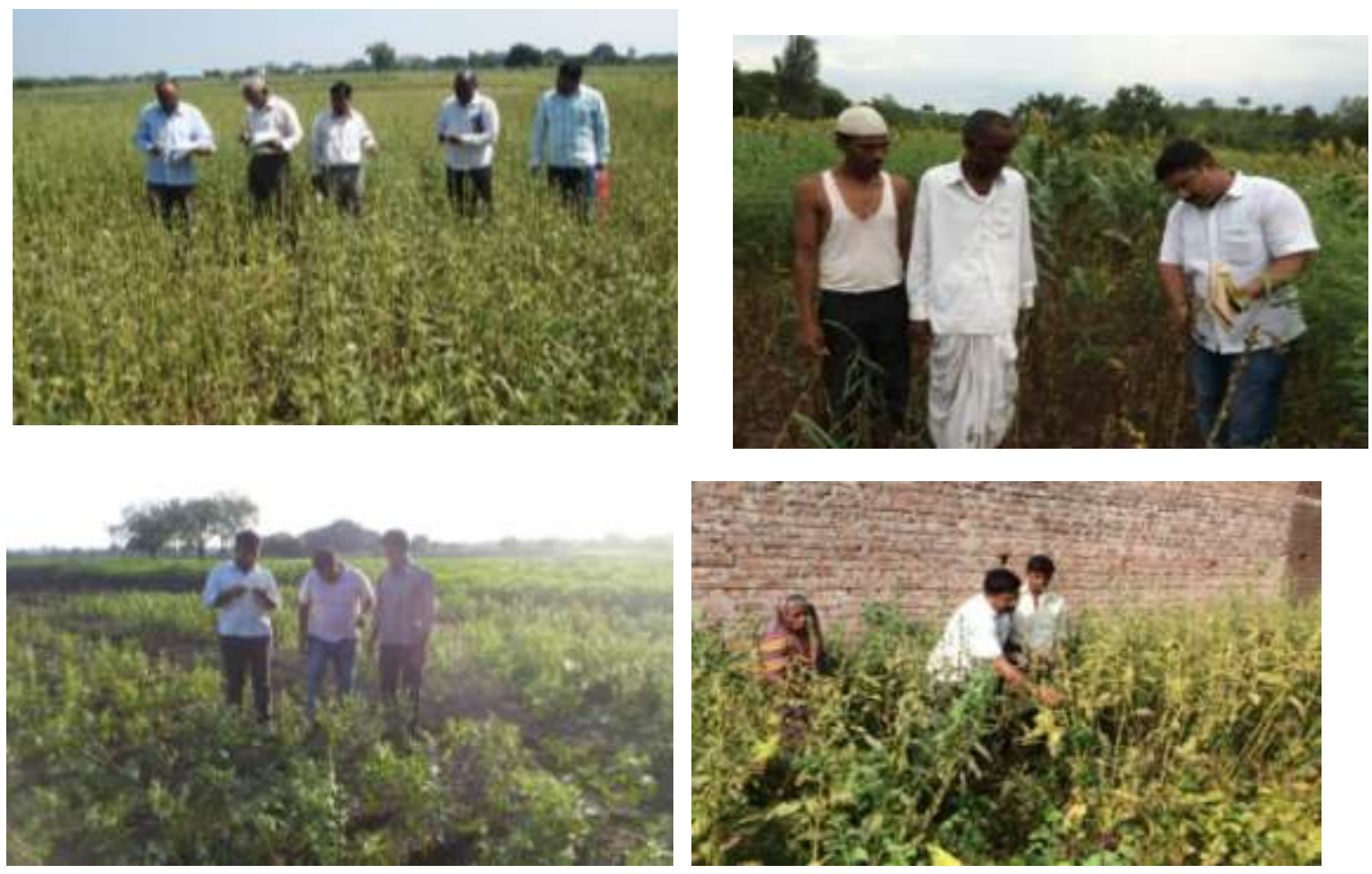

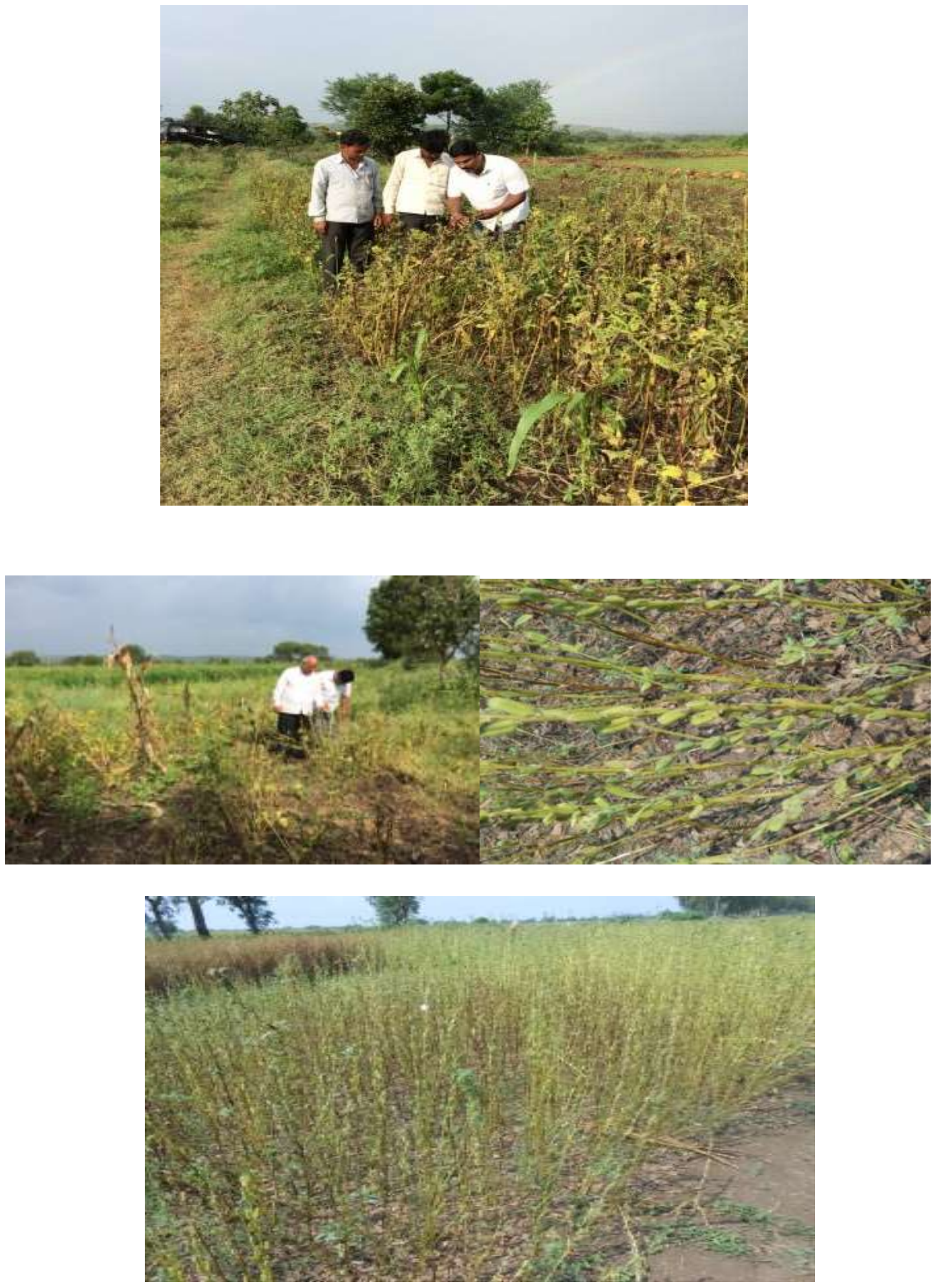

Sesame crop fields surveyed for recording Alternaria blight diseases intensity During Kharif, 2014 - 2015 


\section{Plate II}
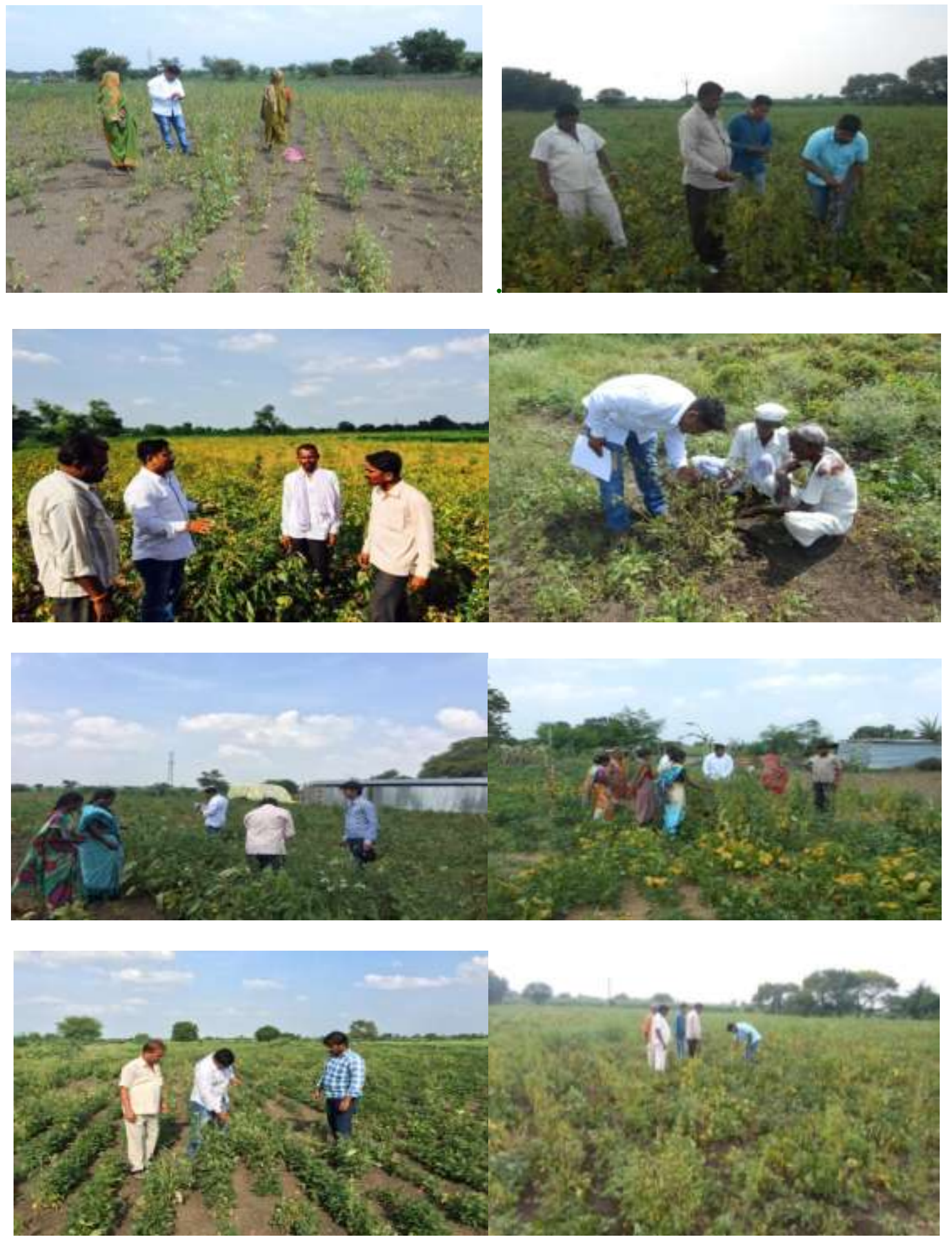

Sesame crop fields surveyed for recording Alternaria blight diseases intensity During Kharif, 2015 - 2016 


\section{Plate III}
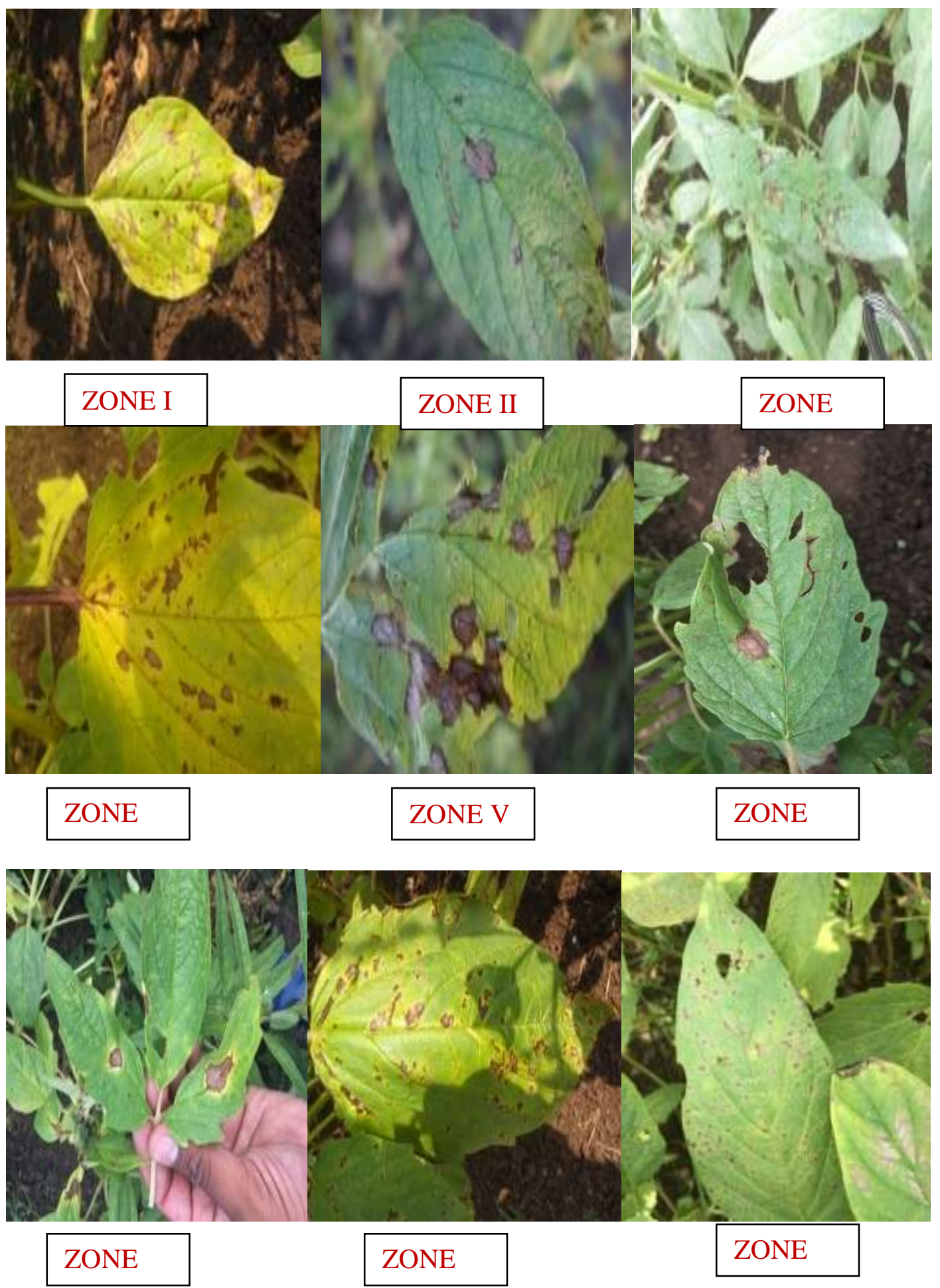

Alternaria blight diseased sesame leaf samples collected during field survey Kharif, 2014 - 2015 


\section{Plate IV}

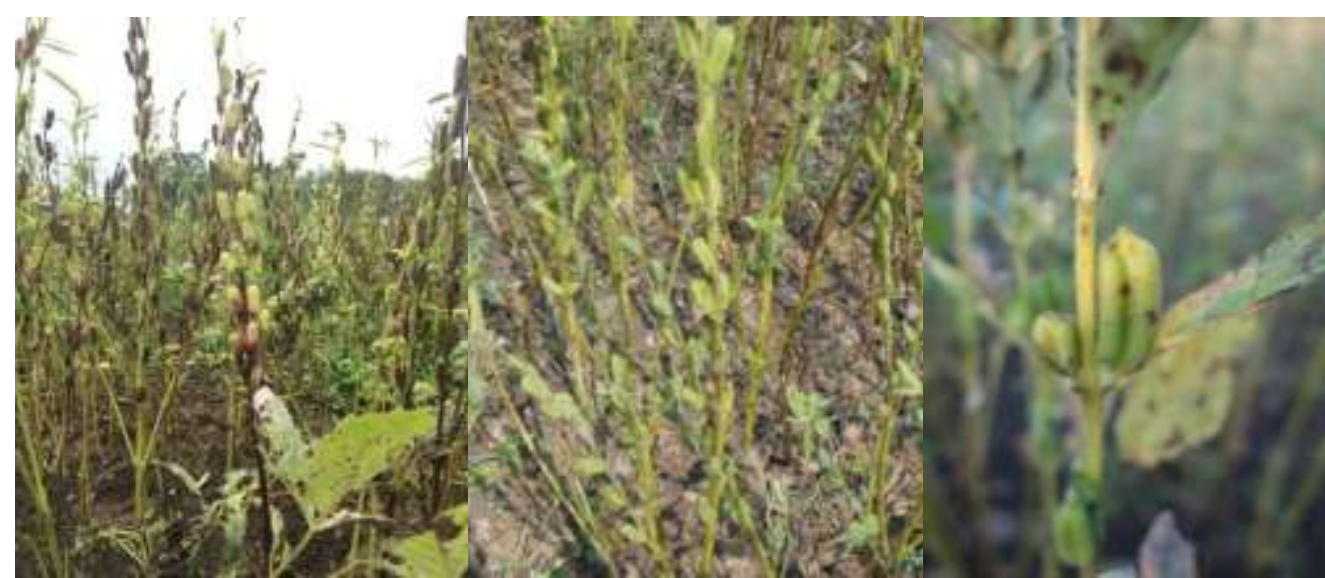

A) Capsule

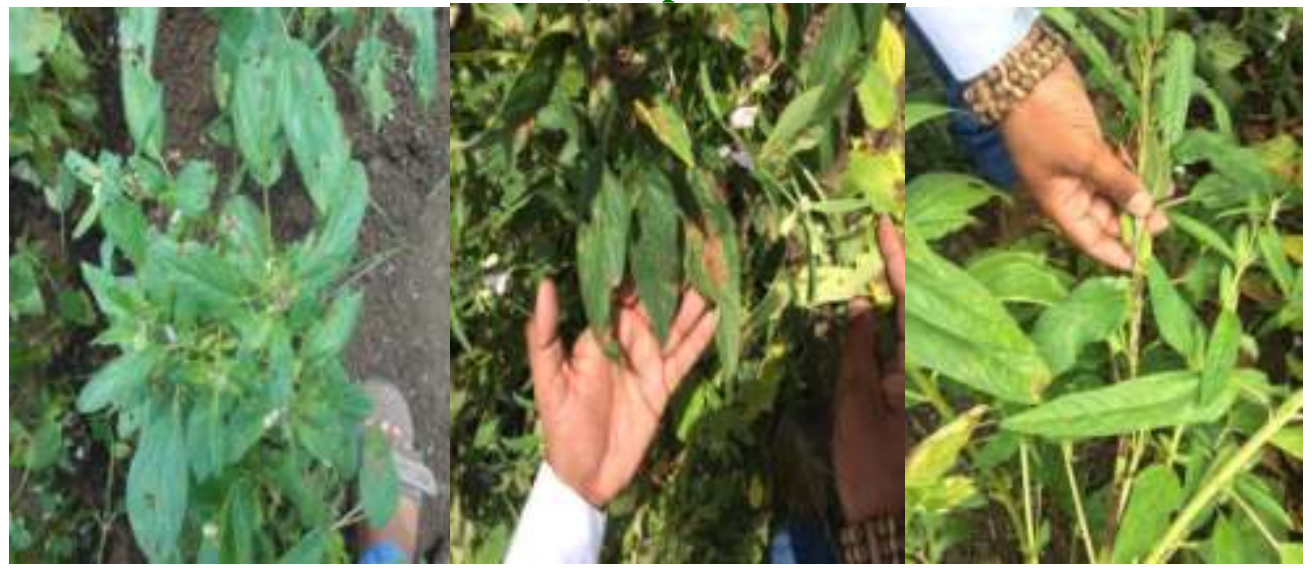

B) Leaf and Stem

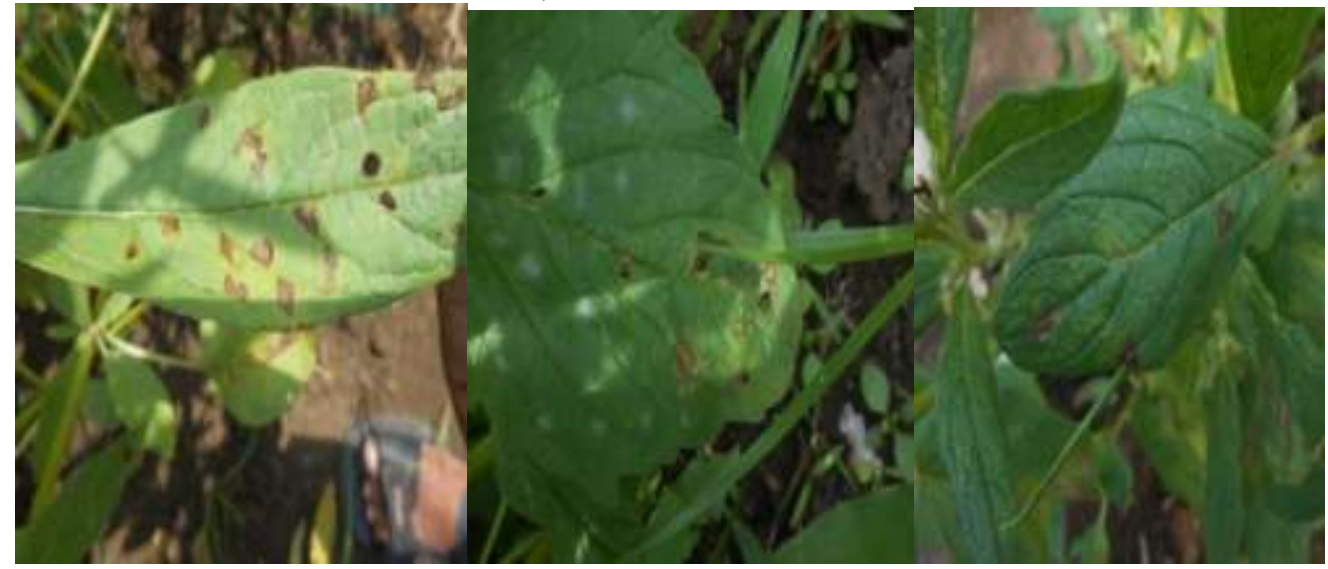

B) Leaf

Typical symptoms of Alternaria blight on sesame crop 


\section{Plate V}

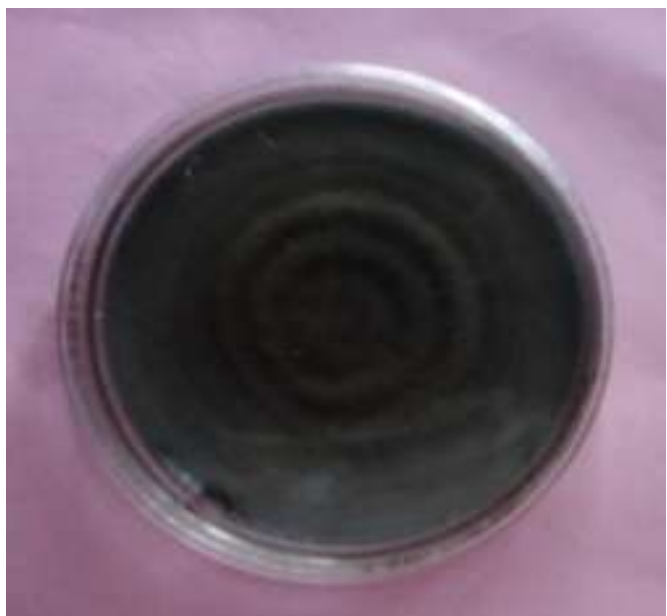

(A)

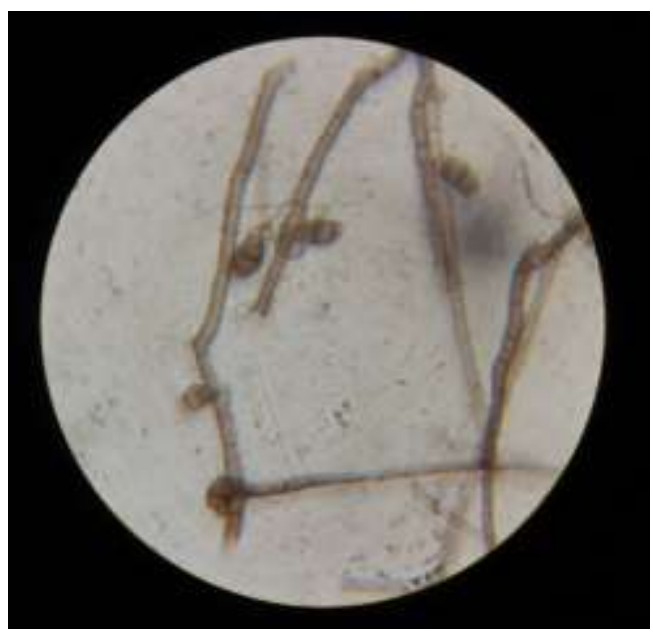

(B)

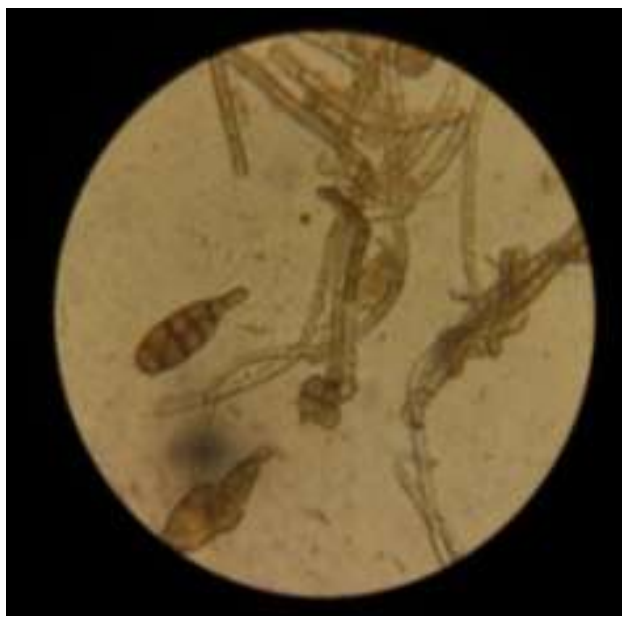

(C)

A. sesami pure culture (A) and microphotograph of its mycelium (B) and conidia (C) 


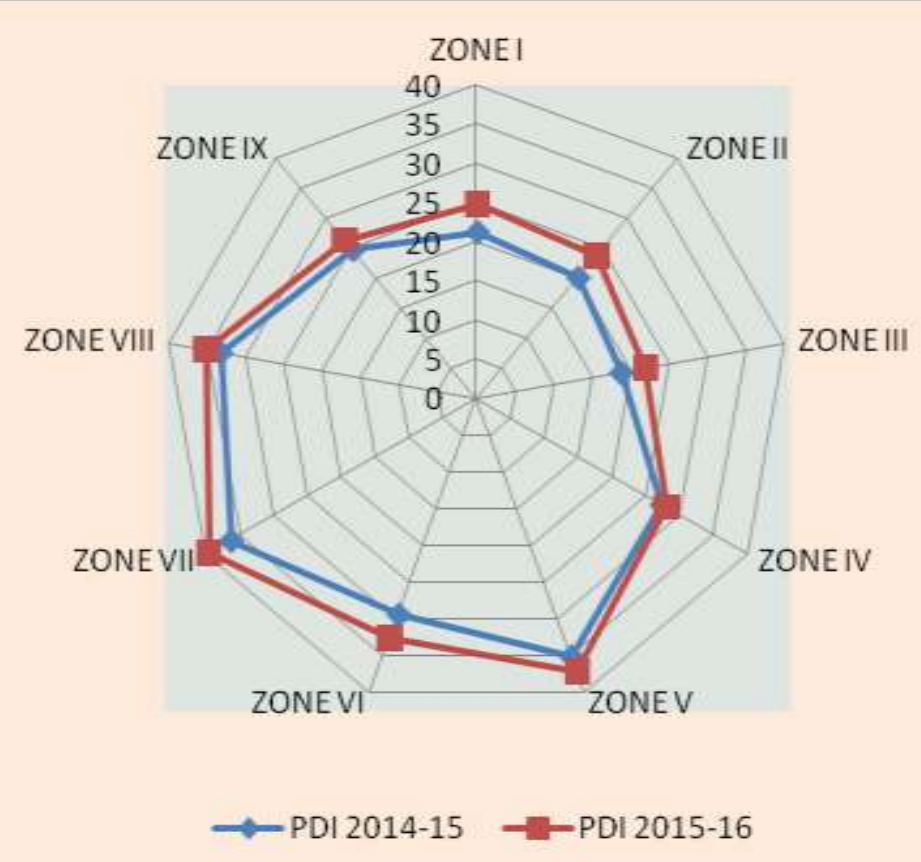

Fig.1 Zone-wise average Alternaria blight intensity on sesame of Maharashtra state during Kharif, 2014-15 and 2015-16

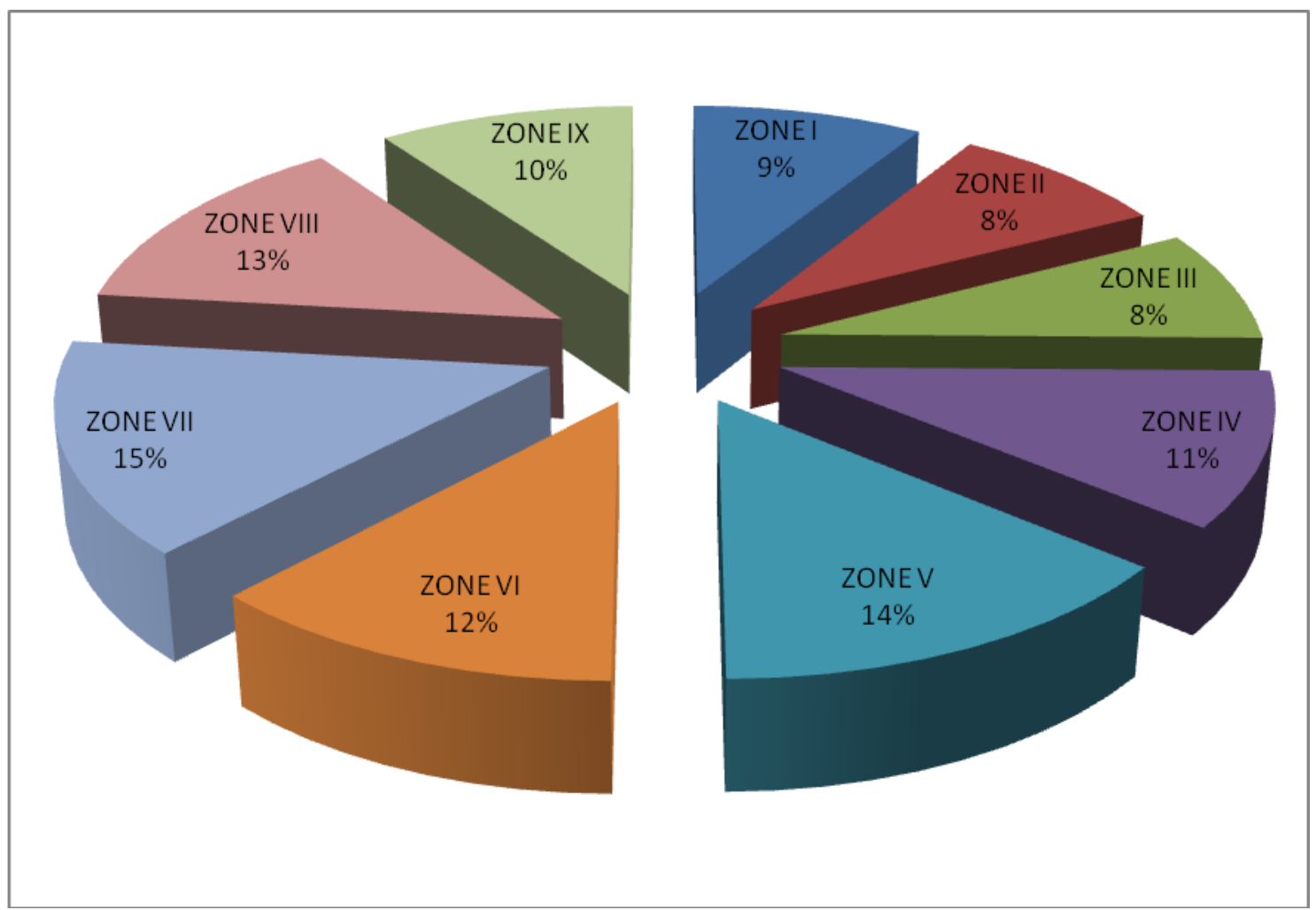

Fig.2 Zone-wise pooled intensity of Alternaria blight on sesame in Maharashtra state (Kharif, 2014-15 and 2015-16) 


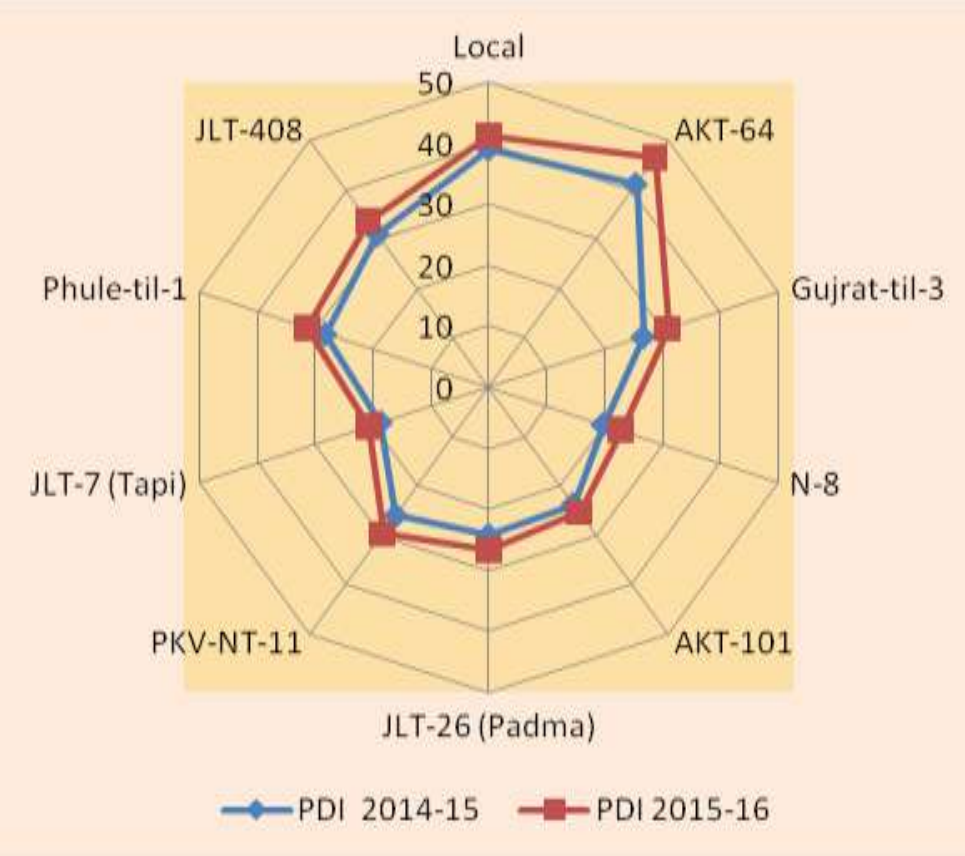

Fig.3 Sesame variety-wise average intensity of Alternaria blight in Maharashtra state during Kharif , 2014-15 and 2015-16

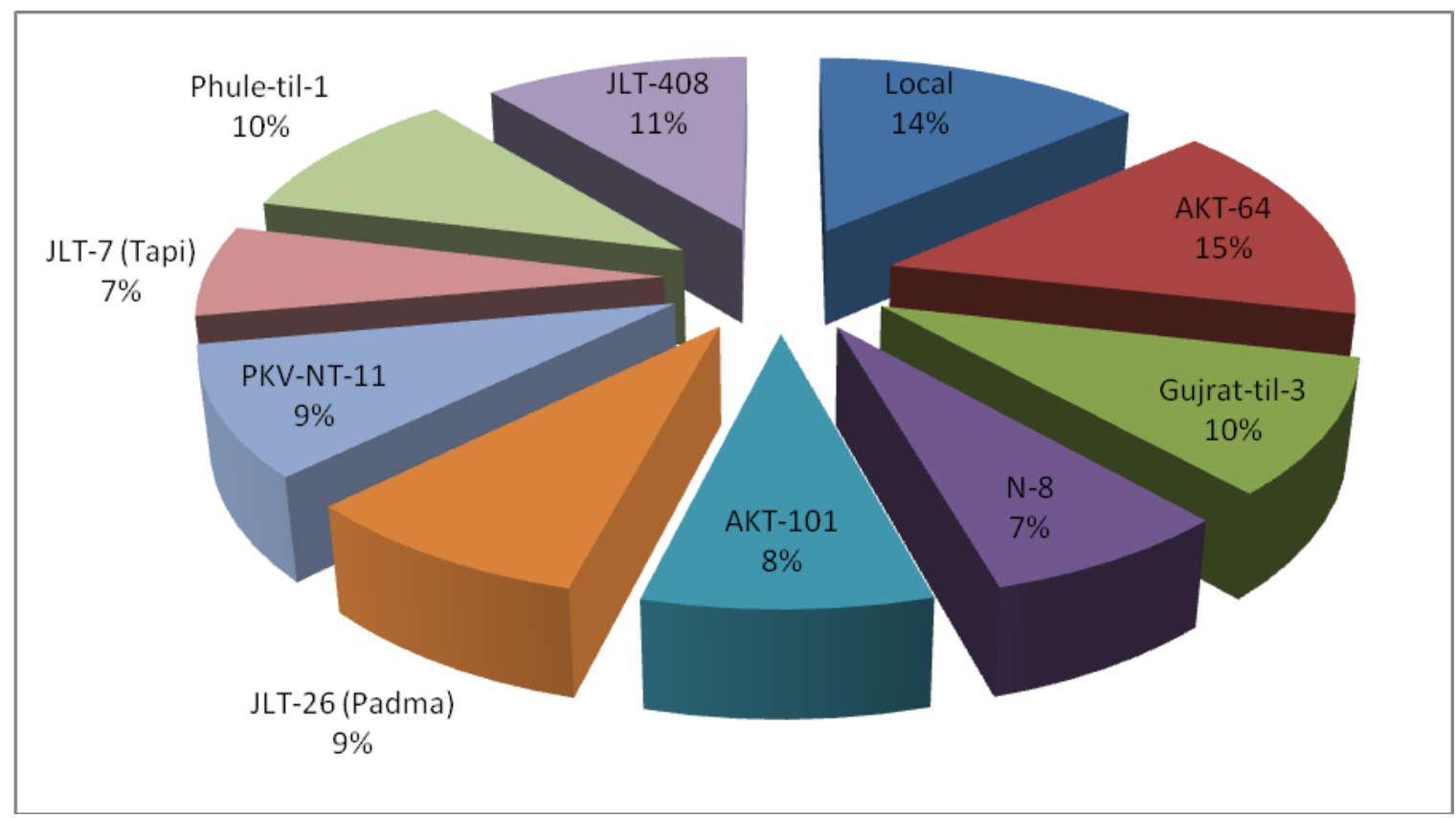

Fig.4 Sesame variety-wise pooled mean intensity of Alternaria blight in Maharashtra state during Kharif, 2014-15 and 2015-16

On the basis of pooled mean (Kharif, 2014-15 and 2015-16) results (Fig. 4), maximum mean blight disease intensity was recorded on
AKT-64 (43.76 \%), followed by Local cultivar (40.08\%); whereas, rest of the cultivars exhibited minimum mean blight 
disease intensity in the range of 19.63 to 32.33 per cent.

\section{Variability in Alternaria blight symptoms}

During survey, natural leaf spot / blight symptoms expressed a wide range of variability in respect of their shape, size, colour and presence or absence of concentric zonation etc. (PLATE III and Table 6).

Shapes of the leaf spots observed were mostly circular to irregular on the sesame crop grown in the four zones viz., North Kokan Coastal Zone, Western Ghat Zone, Sub Mountain Zone and Western Maharashtra Scarcity Zone; circular from the two zones viz., South Kokan Coastal Zone and Central Vidarbha Zone and irregular from the three zones viz., Western Maharashtra Plain Zone, Central Maharashtra Plateau Zone and Eastern Vidarbha Zone.

Size of the leaf spots was also varied and were small from the one zone Western Ghat Zone; medium from the three zones viz., Sub Mountain Zone, Western Maharashtra Scarcity Zone and Central Vidarbha Zone; large from the three zones viz., North Kokan Coastal Zone, Western Maharashtra Plain Zone and Central Maharashtra Plateau Zone and small to large from the two zones viz., South Kokan Coastal Zone and Eastern Vidharbha Zone.

Colour of the leaf spots on sesame crop was mostly light brown to dark brown from the four zones viz., North Kokan Coastal Zone, Sub Mountane Zone, Western Maharashtra Plain Zone and Eastern Vidarbha Zone; dark brown to black from the one zone Central Vidarbha Zone; light brown from the two zones viz., Western GhatZone and Western Maharashtra Scarcity Zone and light gray from the two zones viz., South Kokan Coastal Zone and Central Maharashtra Plateau Zone. Concentric zonations around the leaf spots were found on the sesame crop grown in the four zones viz., South Kokan Coastal Zone, Western Maharashtra Scarcity Zone, Central Vidarbha Zone and Eastern Vidarbha Zone; whereas, there absence of concentric zonation around the leaf spots on sesame crop grown in the five zones viz., North Kokan Coastal Zone, Western Ghat Zone, Sub Mountain Zone, Western Maharashtra Plain Zone and Central Maharashtra Plateau Zone. Thus, during survey, distinguishing features of the leaf spot / blight on sesame crop observed were circular or irregular, medium to large sized, light brown to dark brown coloured leaf spots with or without concentric zonation.

From the ongoing results it has been inferred that the sesame Alternaria blight disease intensity varied at different locations surveyed of nine agro-climatic zones of Maharashtra state falling under various climatic conditions, inoculum potential and varieties cultivated with different genetic makeup. Overall average disease intensity was of higher tune in the range of 21.83 to 39.58 per cent during Kharif, 2015-16 than that of Kharif, 2014-15 (18.64 to $36.39 \%$ ). This may be due to overall maximum rainfall received during rainy season of 2015-16.

These results of the present studies on occurrence and distribution of Alternaria blight disease in sesame crop are similar to those reported earlier by several workers (Mohanty and Behera, 1958; Leepik and Sowell, 1964; Chohan, 1978; Kolte, 1985; Naiket al., 2004; Akbari and Parkhia, 2011; Anonymous, 2014).

Ojiambo et al., (1998) reported the long period of high humidity and spore dispersal by frequent rain showers during Kharif were more suitable for infection of leaf spot / blight of sesame. Leepik and Sowell (1964) reported that species Alternaria sesami probably occurs wherever sesame is grown. Akbari and Parkhia (2011) reported occurrence of 
Alternaria leaf spot / blight of sesame from Saurashtra region of Gujrat state, which caused 80 per cent losses in grain yield under wet climate. Anonymous (2014) reported similar result that Alternaria leaf blight of sesame incidence were higher in Tamil Nadu (Cuddalore district)1-2 grade, Keonjhor and Dhenkanal districts (2-3 grade) and less in Madhya Pradesh (Jabalpur and Chhatrapur districts) 1-2 grade, Jodhpur district (0-2 grade) due to unfavourable environmental conditions prevailed.

\section{References}

Akbari, L. F. and Parakhia, A. M. (2011). Alternaria blight - New outbreak on sesame in Gujarat. J. Mycol. Pl. Pathol. 41(1): 163.

Anonymous (2014). AICRP (Sesamum and Niger), Annual report, (2013-2014) JNKVV Campus, Jabalpur-482004.

Anonymous (2015). Ministry of Agri. Gove. India. (ON 410).

Ashri,A. (1998). Sesame breeding. Plant Breed Reviews. $16: 179-228$.

Balasubramanian, T. N. and S. P. Palemiappan. (2000). Sesame In: P.S. Rathore (ed.) Techniques and Management of Field crop Production. Agrobios (India), Jodhpur. : 178-196.

Bhale, M. S., Bhale, U. and Khare, M. N. (1998).Disease of important oilseed crops and their management. In pathological problems of economic crop plants and their management.(Eds. S.M. Paul Khurana), Scientific Publishers, Jodhpur.: 251-279.

Chohan, J. S. (1978). Diseases of Oilseed crops, future plans and strategy for control under small holdings. Indian Phytopath.31: 1-15.

Dey, P.K. (1948).Plant Pathology Administrative Report of Agriculture Department, Uttar Pradesh, India. : 4346.
Dolle, U. V. 1981 Epidemiology and control of leaf blight of sesame caused by A. sesami (Kawamura). Mahanty and Behera. Plant Pathology News Letter 2: 1-10.

Joshi, A. B. (1961). Sesamum. Indian Central Oilseeds Committee, Hyderabad, India.

Kolte, S. J. (1985). Disease of Annual Edible Oilseed Crops. (Vol.-II): Rapeseed, Mustard, Safflower and Sesame diseases. CRC Press Inc, Boca Raton, Florida, USA.PP-135.

Kumar, P. and Mishra U. S. (1992) Diseases of Sesamum indicum in Rohilkhand: intensity and yield loss. Indian Phytopath. 45 (1): 121-122.

Leppik, E. E. and Sowell, G. (1964). Alternaria sesami a serious seed born pathogen of world wide distribution. FAO Pl. Prot. Bull. 12 (1): 13-16.

Mayee, C. D. and Datar, V. V. (1986). Phytopathomethory: Technical Bulletin Published by Marathwada Agric. Univ., Parbhani (M.S.) India. : 100-104.

McKinney (1923).A new system of grading plant diseases. J. Agric. Res. 26 : 195218.

Mohanty, N. N. and Behera, B. C. (1958). Blight of sesame (Sesame orientale L.) caused by Alternaria sesami (kawanura) n. comb. Current Science 27:492-493.

Naik, M.K., Patil, R.G., Suvarna and Ajithkumar, K. (2004). Yield losses model and blight prediction model in Alternaria blight of sesame. Indian Phytopath. 57: 106 (Abstract).

Narute, T. K., and Utikar, (1994). Efficacy and economics of fungicidal control of Alternaria leaf blight of sesamum. J. Maha. Agric. Sci. 19. PP 449.

Ojiambo, P.S., Narla, R.D., Ayiecho, P.O. and Nyabundi J.O. (1998). Effect of infection level of sesame (Sesamum indicum L.) seed by Alternaria sesami on severity of Alternaria leaf spot. Tropical Agric. Res. and extension. 
1(2): 125-130.

269-303.

Prasad, P. R. and Reddy, S. R. Weiss, E. A. (1983). Oilseed crops, Longman, (1997).Diseases of two districts (Warangal and Karimnagar) of Andhara Pardesh. Microbio. Biotech. : 169-174.

Tripathi, U. K., Mehata, N and Sangwan M. S (2005). Fungal and bacterial diseases of sesame. In: Diseases of oilseed crops (eds.) Indus Pub. New Delhi, India:

London and New York: 660.

Yertmanos, D.M. (1980). Sesame, (In) Hybridization of Crop Plants. W.R Fehr and H.H. Hadley (Eds), American Society of Agronomy, Crop Science Society of America, Madison, Wisconsin. Pp. 549-563.

\section{How to cite this article:}

Pawar, D.V., A.P. Suryawanshi and Kadam, V.A. 2019. Occurrence and Distribution of Sesame Alternaria Leaf Blight Disease in Nine Agro Climatic Zones of Maharashtra State. Int.J.Curr.Microbiol.App.Sci. 8(10): 2326-2343. doi: https://doi.org/10.20546/ijcmas.2019.810.270 